\title{
Fiber movements and sound attenuation in glass wool
}

\author{
Tarnow, Viggo
}

\section{Published in:}

Acoustical Society of America. Journal

Link to article, DOI:

10.1121/1.424581

Publication date:

1999

\section{Document Version}

Publisher's PDF, also known as Version of record

Link back to DTU Orbit

Citation (APA):

Tarnow, V. (1999). Fiber movements and sound attenuation in glass wool. Acoustical Society of America. Journal, 105(1), 234 - 240. https://doi.org/10.1121/1.424581

\section{General rights}

Copyright and moral rights for the publications made accessible in the public portal are retained by the authors and/or other copyright owners and it is a condition of accessing publications that users recognise and abide by the legal requirements associated with these rights.

- Users may download and print one copy of any publication from the public portal for the purpose of private study or research.

- You may not further distribute the material or use it for any profit-making activity or commercial gain

- You may freely distribute the URL identifying the publication in the public portal

If you believe that this document breaches copyright please contact us providing details, and we will remove access to the work immediately and investigate your claim 


\title{
Fiber movements and sound attenuation in glass wool
}

\author{
Viggo Tarnow \\ Department of Applied Engineering Design and Production, Technical University of Denmark, \\ Bygning 358, DK 2800 Lyngby, Denmark
}

(Received 24 December 1997; revised 24 August 1998; accepted 25 September 1998)

\begin{abstract}
Propagation of a plane harmonic sound wave in fiber materials such as glass wool is studied theoretically and experimentally. Wave equations are set up that take into account the movement of the fiber skeleton. The attenuation of the sound wave in slabs of glass wool was calculated and measured. The main new result is that the experimental attenuation of a low-frequency propagating wave is lower when the fibers move. For a wave with frequency $100 \mathrm{~Hz}$ in glass wool of density 30 $\mathrm{kg} / \mathrm{m}^{3}$, the attenuation of a layer of thickness $0.20 \mathrm{~m}$ is $4 \mathrm{~dB}$ if the fibers move, and $12 \mathrm{~dB}$ if they do not move. The attenuation was computed from the fiber diameters and their density, which was found from the mass density. Measured attenuation is lower than the values calculated. Nevertheless, if the density is adjusted, a complete fit is obtained between experimental and theoretical values for frequencies 50-5000 Hz. (C) 1999 Acoustical Society of America.

[S0001-4966(99)02601-6]

PACS numbers: 43.58.Vb, 43.20.Jr, 43.55.Ev, 43.35.Mr [SLE]
\end{abstract}

\section{INTRODUCTION}

In theories that aim at computing the sound attenuation of fiber materials, one often assumes that the fibers do not move. However, several people have measured the effect of the movements of fibers.

Dahl et al. ${ }^{1}$ measured the absorption coefficient of Kevlar samples with a thickness of $10 \mathrm{~cm}$ placed on a hard wall. They found a resonance in the absorption attributed to a mechanical resonance due to movement of fibers. The absorption was measured for densities $5-67 \mathrm{~kg} / \mathrm{m}^{3}$, and it was found that resonances were sharpest for the highest-density material, where the resonance frequency was about $600 \mathrm{~Hz}$.

Allard et al. ${ }^{2}$ measured the surface impedance of samples of glass wool with density $130 \mathrm{~kg} / \mathrm{m}^{3}$ attached to an impervious floor. They measured a resonance frequency of $470 \mathrm{~Hz}$ for a layer of thickness $10 \mathrm{~cm}$, and $860 \mathrm{~Hz}$ for thickness $5.4 \mathrm{~cm}$. These resonances were attributed to mechanical resonance of the samples due to the movements of fibers.

Lambert ${ }^{3}$ reported measurements on Kevlar samples of thickness $10 \mathrm{~cm}$ placed on a hard wall. A resonance about $1000 \mathrm{~Hz}$ was found in the attenuation, the phase speed, the characteristic admittance of the material, the effective resistivity, and the effective dynamic density. These resonances were caused by moving fibers. In a second paper $^{4}$ Lambert gave further data for resonances in the effective resistivity and the effective dynamic density.

In the present work, measurements of the sound attenuation of glass wool are reported, which show that at frequencies below $200 \mathrm{~Hz}$ the fiber movement strongly influences the attenuation. The measurements were done on glass wool slabs with density 14 and $30 \mathrm{~kg} / \mathrm{m}^{3}$. The glass wool slabs had dimensions $100 \times 600 \times 900 \mathrm{~mm}$ and were placed in a stack of six units, and placed in a box made of chipboards open only at one side.

Several theories have been published that take into account the movements of the skeleton of porous materials. Biot published two papers ${ }^{5,6}$ in which a porous solid filled with a fluid is studied.
The papers by Dahl et al. ${ }^{1}$ and Lambert ${ }^{3,4}$ explain the measured acoustic properties by resonances in single fibers. However, it is difficult to see how single fiber could resonate, because all fibers are strongly coupled, and the wavelength of a sound wave are much longer than the distance between the fibers. The theoretical model in the present paper does not use resonating single fibers.

In the paper by Allard, ${ }^{2}$ the observed resonances were explained by the methods of the two Biot papers. ${ }^{5,6}$ A similar approach was used in the paper by Johansen, Allard, and Brouard, ${ }^{7}$ who used finite element methods to analyze acoustical measurements on fiber material samples placed in a tube with diameter $10 \mathrm{~cm}$. Two thicknesses, 2 and $5 \mathrm{~cm}$, were used. They found resonances due to fiber movements could be explained by assuming the skeleton moved as a continuous medium.

In the present paper the sound attenuation is computed, allowing for the fiber movements by regarding the skeleton as a continuous medium in the same way as Biot did. The dynamic resistivity and compressibility used here are computed more nearly accurately than the ones used by Biot.

The earlier reports by Allard $e t a l .^{2}$ and Johansen $e t a .^{7}$ of observations of fiber movement used mechanical resonance in the samples to detect the movements of fibers. This is not so in this paper where the attenuation of propagating waves in glass wool is measured. The paper by Johansen, Allard, and Brouard ${ }^{7}$ referred to measurements on samples with cylindrical shape and a diameter of $10 \mathrm{~cm}$. They had to take into consideration the lateral movement of the fiber skeleton. This is not necessary for the measurements reported in this paper, because the samples used here are much wider. The dimensions are $10 \mathrm{~cm} \times 60 \mathrm{~cm} \times 90 \mathrm{~cm}$.

\section{THEORY OF SOUND PROPAGATION WITH FIBER MOVEMENTS}

In fiber materials, such as glass wool, a coupling between movements of air and fibers exist; this coupling is mainly due to friction between air and fibers. Wavelengths of 
audible sounds in air and fibers are much longer than the distance between fibers in ordinary glass wools. Therefore, the propagation of sound waves can be calculated by assuming the medium is continuous, and the waves cannot "see" the single fibers.

\section{A. Elastic forces}

We study plane waves in glass wool that propagate in the $x$ direction of a rectangular coordinate system. The displacement of the fiber skeleton in the $x$ direction is called $u$, and the mean displacement of air in the $x$ direction is called $\nu$. It is defined as the volume flow of air through a large cross section divided by the area of that cross section. [Biot ${ }^{5}$ used another definition of mean air displacement: Volume flow of fluid (air) divided by area of fluid in that cross section. He assumed "that the solid-fluid system is statistically isotropic." Glass wool is anisotropic, and for some boundary conditions, one needs the air volume flow through a cross section. This is, with our definition, simply equal to mean air displacement times the area of the cross section. However, this is not so with Biot's definition.]

The elastic forces on fibers and air can be found from the potential energy per unit volume. The potential energy density $E$ is, in a linear theory, a quadratic form in the partial derivatives of the displacement of the fibers $u_{x}$ and air $\nu_{x}$. Thus

$$
E=\frac{1}{2} c_{11} u_{x}^{2}+A u_{x} \nu_{x}+\frac{1}{2} K \nu_{x}^{2},
$$

where $c_{11}$ is an elastic modulus of the fiber skeleton, when the air is not displaced $\left(\nu_{x}=0\right) ; K$ is the bulk modulus of air, when the fibers do not move $\left(u_{x}=0\right) ; A$ is a constant that couples movements of air and fibers.

The static value of $c_{11}$ was measured by applying a known force to a sample of the glass wool and measuring the depression. $c_{11}$ equals the force per area divided by the relative depression. The result was $c_{11}=2000$ and $16000 \mathrm{~Pa}$ for the two glass wool types. We regard the glass fiber skeleton as a space lattice of glass fibers. They may vibrate, but the fibers are coupled. This system is similar to atoms in a crystal, the atoms are placed in a space lattice, they vibrate, but their vibrations are coupled. The dynamics of the space lattice show that the elastic moduli does not depend on frequency when the wavelength is longer than the distance between the fibers. Therefore, we assume the elastic modulus $c_{11}$ does not depend on frequency. Measurements of the elastic moduli for glass wool as a function of frequency have not been published.

The bulk modulus of air equals

$$
K=\frac{1}{\phi C},
$$

where $\phi$ is the porosity, and $C$ is the dynamic compressibility of air itself, which will be computed by the self-consistent method of Tarnow. ${ }^{8}$ Self-consistent methods were first used in atomic physics, Schiff. ${ }^{9}$ A model with parallel, randomly placed cylinders (fibers) with equal diameters will be used. The procedure is described in Appendix A.

The value of $A$ can be found by computing the pressure $p$ from Eq. (1). This gives

$$
-p=\frac{\partial E}{\partial \nu_{x}}=A u_{x}+K \nu_{x} .
$$

If $p=0$ and the fibers move out of a volume, some air must flow into that volume to keep the air pressure zero. The volume of fibers going out of a unit volume of space is $d u_{x}$, where $d$ is the volume density of fibers, $d=1-\phi$. They are practically incompressible compared to air; therefore, the volume of air that streams out of a unit volume must be

$$
\nu_{x}=-d u_{x} .
$$

From Eq. (3) $(p=0)$ and Eq. (4), one gets

$$
A=d K \text {. }
$$

From Eqs. (1) and (5), the elastic energy density becomes

$$
E=\frac{1}{2} c_{11} u_{x}^{2}+d K u_{x} \nu_{x}+\frac{1}{2} K \nu_{x}^{2} .
$$

The stress $\sigma$ in the fiber lattice can also be found from the elastic energy density, Eq. (6):

$$
\sigma=\frac{\partial E}{\partial u_{x}}=c_{11} u_{x}+d K \nu_{x} .
$$

From this equation one sees that the elastic modulus $c_{11}$ should be measured by constant air volume $\left(\nu_{x}=0\right)$. However, it was measured by constant pressure. The relation between the two moduli can be found by setting Eq. (4) into Eq. (7). Thus

$$
\sigma=c_{11} u_{x}-d^{2} K u_{x} .
$$

From the last equation, one finds the relation between the elastic modulus for constant air pressure and volume $c_{p 11}$ and $c_{\nu 11}$. The result is

$$
c_{p 11}=c_{\nu 11}-d^{2} K .
$$

However, the difference between the two elastic moduli is small, $2 \%$ and $1 \%$ for $d=0.0056$ and 0.016 , which corresponds to mass density 14 and $30 \mathrm{~kg} / \mathrm{m}^{3}$.

\section{B. Viscous and inertial forces}

We assume a harmonic time variation, described by a complex time factor $e^{-i \omega t}$, where $\omega$ is the cyclic frequency and $t$ is the time. In an earlier paper ${ }^{10}$ by the author, the forces on the fibers from the air stream are computed when the fibers do not move. The movement of the fibers can be taken care of by changing the boundary condition on the fibers, and repeating the calculation in the paper. ${ }^{10}$ When this is done, one finds the forces $f_{f}$ on the fibers in a unit volume of glass wool,

$$
f_{f}=-d \rho \omega^{2} u+R_{\perp}^{\prime} i \omega(u-\nu),
$$

where $\rho$ is the mass density of air. $R_{\perp}^{\prime}$ is the dynamic resistivity of the fibers, which will be computed by the selfconsistent method of the paper in Ref. 10, summarized in Appendix B.

Equation (10) can be controlled in the high-frequency limit. It follows from the article in Ref. 10 that the highfrequency limit of the resistivity is

$$
R_{\perp}^{\prime} \rightarrow-2 d \rho i \omega .
$$


When this is set into Eq. (10), one obtains

$$
f_{f}=d \rho \omega^{2} u-2 d \rho \omega^{2} \nu .
$$

This is reasonable because the inertia of a cylinder oscillating perpendicular to its axis in a fluid is increased by the mass of fluid displaced by the cylinder, Lamb. ${ }^{11}$ Equation (12) is also according to Eq. (11.9) in Sec. 12 of Landau and Lifschitz. ${ }^{12}$ They assumed potential flow, which is valid for high frequencies, where the viscous boundary layer is very thin.

The viscous forces on the air follow from Newton's law of action and reaction. Therefore, the force on the air in a unit volume is

$$
f_{a}=-R_{\perp}^{\prime} i \omega(u-\nu) .
$$

This can be checked in the high-frequency limit. From Eqs. (11) and (13) one gets, in this limit,

$$
f_{a}=2 d \rho \omega^{2} \nu-2 d \rho \omega^{2} u .
$$

By considering only inertial forces, and using Newton's second law $f_{a}=-\rho \omega^{2} \nu$. This is set into Eq. (14), and one gets

$$
-(1+2 d) \rho \omega^{2} \nu=-2 d \rho \omega^{2} u .
$$

Rayleigh $^{13}$ computed the effective mass $\rho_{\text {eff }}$ for potential flow perpendicular to fixed cylinders. He found

$$
\rho_{\mathrm{eff}}=\frac{1+d}{1-d} \rho .
$$

To first order in $d$, this is $\rho_{\text {eff }}=(1+2 d) \rho$, which agrees with the left side of Eq. (15).

\section{Wave equations}

The equations for a plane wave propagating in the $x$ direction of a rectangular coordinate system can be found by applying Newton's second law to fibers and air in a unit volume. By computing the forces on a unit volume from the density of elastic energy, Eq. (6), and the viscous forces, Eqs. (10) and (13), one gets

$$
\begin{aligned}
-\omega^{2}\left[\begin{array}{cc}
\mu-d \rho & 0 \\
0 & \rho
\end{array}\right]\left[\begin{array}{l}
u \\
\nu
\end{array}\right]= & {\left[\begin{array}{cc}
c_{11} & d K \\
d K & K
\end{array}\right]\left[\begin{array}{c}
u_{x x} \\
\nu_{x x}
\end{array}\right] } \\
& +i \omega R_{\perp}^{\prime}\left[\begin{array}{cc}
1 & -1 \\
-1 & 1
\end{array}\right]\left[\begin{array}{c}
u \\
\nu
\end{array}\right],
\end{aligned}
$$

where $\mu$ is the ordinary mass density of glass wool, and $u_{x x}$ and $\nu_{x x}$ are the second partial derivatives of the displacement of the fiber and air.

\section{Solution of wave equations}

We assume plane wave solutions to Eq. (17) of the form

$$
\left[\begin{array}{c}
u \\
\nu
\end{array}\right]=\left[\begin{array}{c}
u_{0} \\
\nu_{0}
\end{array}\right] e^{i k x},
$$

where $u_{0}$ and $\nu_{0}$ are constants, and $k$ is a wave number. This equation is substituted in Eq. (17). A nontrivial solution exists if the following determinant is zero:

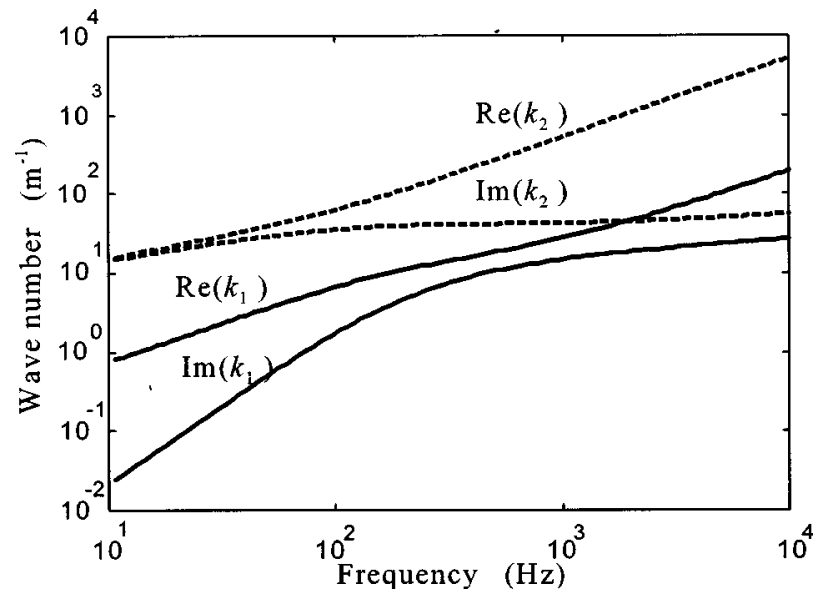

FIG. 1. Wave numbers computed for glass wool of density $14 \mathrm{~kg} / \mathrm{m}^{3}$, volume density 0.0056 , diameter of fibers $6.8 \mu \mathrm{m}$, and elastic modulus 2000 $\mathrm{Pa}$. The waves associated with $k_{1}$ carry most of the sound pressure, the ones associated with $k_{2}$ carry most of the mechanical stress of the fiber skeleton. The real part of the wave number gives the phase shift per meter, and the imaginary part the relative attenuation per meter.

$$
\left|\begin{array}{cc}
-\omega^{2}(\mu-d \rho)+k^{2} c_{11} & k^{2} d K-i \omega R_{\perp}^{\prime} \\
k^{2} d K-i \omega R_{\perp}^{\prime} & -\omega^{2} \rho+k^{2} K
\end{array}\right|=0 .
$$

This equation has two solutions $k_{1}$ and $k_{2}$, which are shown in Fig. 1 as a function of frequency. The curves were computed with a fiber diameter $6.8 \mu \mathrm{m}$, the distance between fibers $80 \mu \mathrm{m}$, volume density $d=0.0056$, and elastic modulus $2000 \mathrm{~Pa}$; which parameters are valid for light glass wool of density $14 \mathrm{~kg} / \mathrm{m}^{3}$. Figure 2 shows the result for glass wool with the same fiber diameter, distance between fibers $58 \mu \mathrm{m}$, fiber density $d=0.016$, elastic modulus $16000 \mathrm{~Pa}$, and mass density $30 \mathrm{~kg} / \mathrm{m}^{3}$.

The constants $u_{0}$ and $\nu_{0}$ of Eq. (18) are related by

$$
\left[\begin{array}{cc}
-\omega^{2}(\mu-d \rho)+k^{2} c_{11} & k^{2} d K-i \omega R_{\perp}^{\prime} \\
k^{2} d K-i \omega R_{\perp}^{\prime} & -\omega^{2} \rho+k^{2} K
\end{array}\right]\left[\begin{array}{c}
u_{0} \\
\nu_{0}
\end{array}\right]=\left[\begin{array}{l}
0 \\
0
\end{array}\right] .
$$

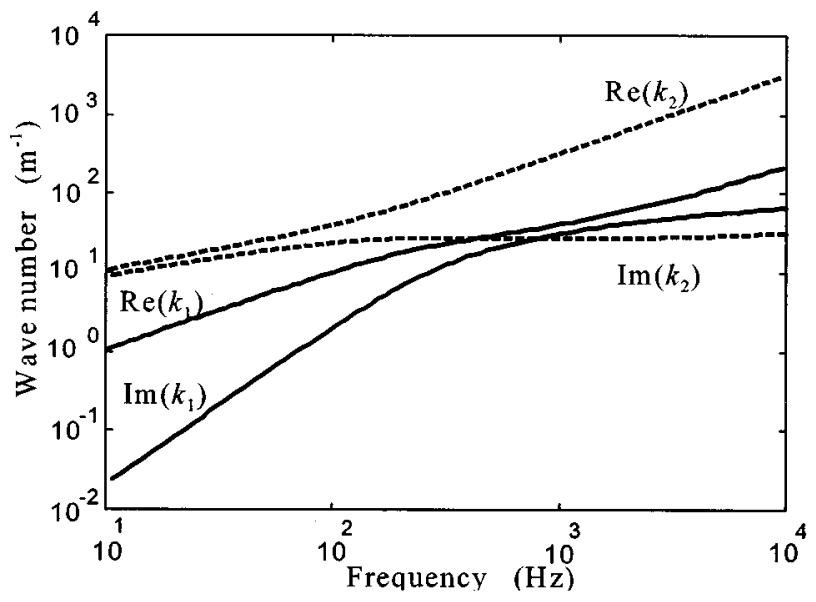

FIG. 2. Wave numbers computed for glass wool of density $30 \mathrm{~kg} / \mathrm{m}^{3}$, volume density 0.016 , diameter of fibers $6.8 \mu \mathrm{m}$, and elastic modulus 16000 $\mathrm{Pa}$. 


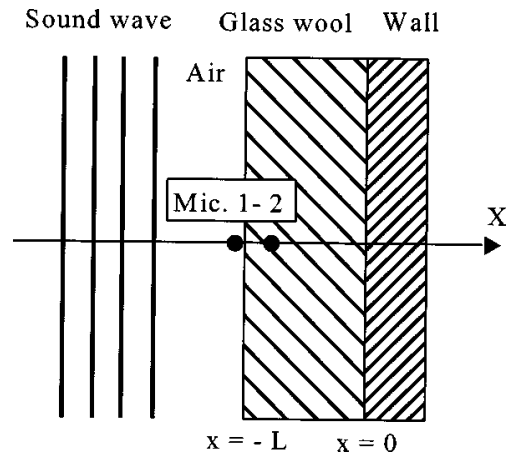

FIG. 3. Plane waves are coming in from the left. They propagate in the glass wool and are reflected by the hard wall. The pressure is measured at the surface of the glass wool and inside it.

In Eq. (20) $k=k_{1}$ or $k_{2}$, the two solutions of Eq. (19). Formulas for $k_{1}$ and $k_{2}$ are in Appendix C.

\section{REFLECTIONS FROM THE REAR}

In the measurements, a sound wave from a loudspeaker reaches a slab of glass wool, the rear side of which is placed on a wall. The wave front is parallel to the slab, as shown in Fig. 3. The sound pressure is measured inside the glass wool. Therefore, we compute the sound pressure of a plane wave inside an infinite slab, which is attached to a hard airtight wall and free on the other side.

The displacement of air particles and the fiber skeleton is zero at the wall. A coordinate system is chosen with an $x$ axis perpendicular to the wall and the origin at the wall. The sound particle displacement $\nu$ can be written as

$$
\nu=\nu_{1} \sin \left(k_{1} x\right)+\nu_{2} \sin \left(k_{2} x\right) .
$$

The constants $\nu_{1}$ and $\nu_{2}$ will be determined by the boundary condition on the free surface of the slab. The displacement of the fiber skeleton $u$ can be written in a similar way,

$$
u=u_{1} \sin \left(k_{1} x\right)+u_{2} \sin \left(k_{2} x\right)
$$

here are $u_{1}$ and $u_{2}$ constants to be determined by the boundary condition on the free surface of the slab.

The slab is placed between $x=-L$ and $x=0$. At the free surface the stress on the fibers is zero. The boundary condition for the derivative of $u$ and $\nu$ can be found from Eq. (7), but because the volume density of fibers is small, one gets

$$
\left[\frac{d u}{d x}\right]_{x=-L}=0
$$

On the free surface the sound pressure $p_{0}$ is given, from Eqs.

(2) and (3) one gets because $d$ is small,

$$
\left[\frac{d \nu}{d x}\right]_{x=-L}=-\phi C p_{0} .
$$

Equations (20), (23), and (24) determine the constants $u_{1}$, $u_{2}, \nu_{1}, \nu_{2}$. The constants $u_{1}$ and $\nu_{1}$ are connected by Eq. (20), where they shall be substituted for $u_{0}$ and $\nu_{0}$. The other two constants $u_{2}$ and $\nu_{2}$ are treated in the same way. The air pressure inside the glass wool can be found from

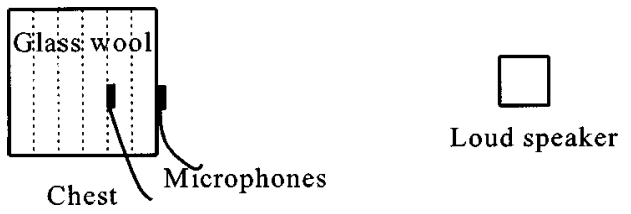

FIG. 4. The measuring setup is placed in an anechoic room. The chest is made of 22-mm thick chipboard with inside dimensions $0.60 \mathrm{~m} \times 0.60 \mathrm{~m}$ $\times 0.90 \mathrm{~m}$. Six slabs 0.10 -m thick of glass wool were placed in it. The chest is open toward the loudspeaker. The two condenser microphones are $\frac{1}{2}$ in. in diameter, and their axes are perpendicular to the direction to the loudspeaker. The distance from the chest to the loudspeaker is $1.70 \mathrm{~m}$.

$$
p=-\frac{1}{\phi C} \frac{d \nu}{d x} .
$$

A formula for the pressure is given in Appendix C.

\section{MEASUREMENT SETUP AND RESULTS}

The attenuation of plane harmonic sound waves propagating in glass wool was measured. Figure 4 shows the setup, which was placed in an anechoic room. A rectangular chest made of $22 \mathrm{~mm}$ thick chipboard was used. It had one open side, which faced a loudspeaker $1.7 \mathrm{~m}$ away. In the chest, six slabs of glass wool each measuring $0.6 \mathrm{~m}$ $\times 0.9 \mathrm{~m} \times 0.1 \mathrm{~m}$ were laid, with fibers parallel to the open side. Two half-inch condenser microphones were used. One was set at the free surface of the glass wool and another was placed inside the glass wool. The axes of the microphones were parallel to the free surface.

The loudspeaker was connected to a sinusoidal voltage supply, the frequency of which could be swept through a range. Attenuation of sound waves was calculated by dividing the pressure at the surface by the pressure inside the glass wool. Measurements were done with the inside microphone $0.2 \mathrm{~m}$ from the free surface and placed in the middle.

Pressure sensitivities of the microphones are equal. At high frequencies, diffraction of waves about the microphone cause changes in the sensitivity. But because frequencies were always below $10 \mathrm{kHz}$, and the direction of propagation perpendicular to the axis of the microphone, this effect is smaller than $0.5 \mathrm{~dB}$ in free air. At $10 \mathrm{kHz}$ the wavelength in the free air is almost equal to the one inside the glass wool. Therefore, the diffraction is small and equal for the two microphones; and has no influence on the measured attenuation.

The inside microphone is set between two glass wool slabs with thickness $100 \mathrm{~mm}$; and the microphone cable is placed between the two slabs that touch each other. The glass wool is soft so there is some deformation of it in a small volume about the microphone. The diameter of this deformation is about $25 \mathrm{~mm}$. Considering the thickness of the glass wool slabs and the lateral dimensions of the slabs, it can be assumed that the pressure measured by the microphone is close to the undisturbed pressure in the glass wool.

The volume density of fibers $d$ equals the mass density of glass wool divided by the mass density of glass, which is $2550 \mathrm{~kg} / \mathrm{m}^{3}$. For glass wools of mass density 14 and 30 $\mathrm{kg} / \mathrm{m}^{3}$, one finds $d=0.0056$ and 0.016 .

To compute the compressibility and resistivity of air as a function of frequency, one must know the radius of fibers $a$ 


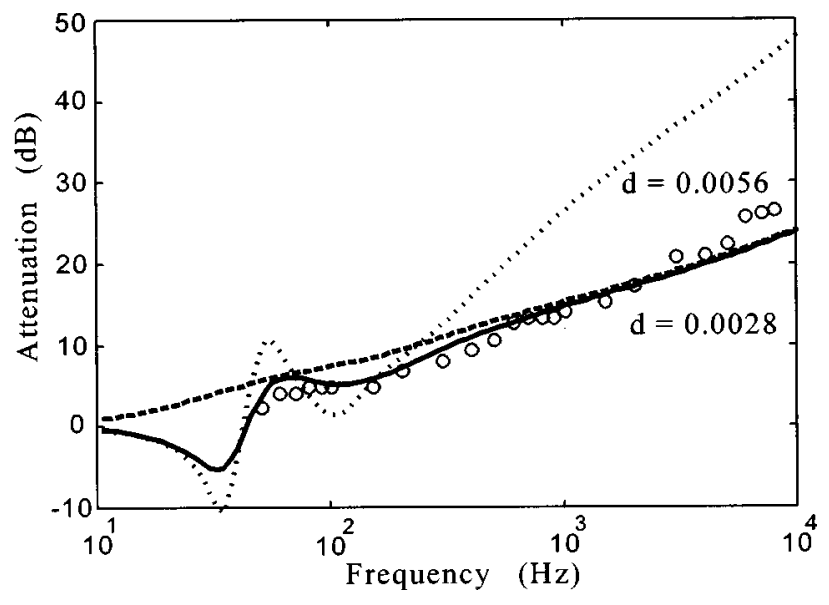

FIG. 5. Measured and computed attenuation of plane sound waves in glass wool of density $14 \mathrm{~kg} / \mathrm{m}^{3}$ and elastic modulus $2000 \mathrm{~Pa}$. The fibers are parallel to the wave front. The attenuation was found $0.20 \mathrm{~m}$ inside the glass wool sample of thickness $0.60 \mathrm{~m}$. The dotted line is computed for a volume density of fibers of 0.0056 and fiber diameter $6.8 \mu \mathrm{m}$. The circles are measurements. The full line is computed for a density of 0.0028 . The dashed line is calculated with the same density but assuming the fibers do not move.

and the distance $b$ between fibers. The diameter of the fibers was measured by a microscope to $6.8 \mu \mathrm{m}$ with a standard variation of $2.7 \mu \mathrm{m}$. The mean distance $b$ is the square root of the area per fiber. It can be calculated from the volume density of fibers $d, b=a \sqrt{\pi / d}$, because we assume parallel fibers. One finds $b=80$ and $58 \mu \mathrm{m}$ for low- and high-density glass wool.

A theoretical calculation of attenuation with moving fibers requires the knowledge of the elastic moduli of the glass wool skeleton. It was measured by turning the chest so that the open side was up. A 22-mm thick chipboard plate with a clearance of $10 \mathrm{~mm}$ along the edge was put on the glass wool and loaded with weights. Depression of the plate was measured as a function of the weight placed on the chipboard plate. The elastic modulus equals the gravity force per area of the plate divided by the corresponding depression. For glass wool of density 14 and $30 \mathrm{~kg} / \mathrm{m}^{3}$, the elastic moduli were measured to be 2000 and $16000 \mathrm{~Pa}$.

The attenuation of sound waves by $0.20 \mathrm{~m}$ glass wool of density $14 \mathrm{~kg} / \mathrm{m}^{3}$ in a slab with a thickness of $0.60 \mathrm{~m}$ is shown in Fig. 5. The dotted line shows the computed pressure for a fiber volume density of 0.0056 , fiber diameter 6.8 $\mu \mathrm{m}$, and elastic modulus $2000 \mathrm{~Pa}$. The measured attenuation is shown as circles. The full line is computed in the same way as the dotted one, but the density of fibers 0.0028 was chosen to make the curve fit the measuring points. The dashed line was computed from the same density, but it was assumed that the fibers did not move.

Figure 6 shows the attenuation for the same depth, thickness of slab, and fiber diameter, but the mass density is 30 $\mathrm{kg} / \mathrm{m}^{3}$. For the dotted line the volume density is 0.016 , fiber diameter $6.8 \mu \mathrm{m}$, and elastic modulus $16000 \mathrm{~Pa}$. The circles are the measured attenuation. It is greater than in Fig. 5, because the mass density is higher. The full line is the calculated attenuation, where the density 0.006 was chosen to fit the curve to the measured points. The dashed line is the calculated attenuation for fixed fibers with the same density.

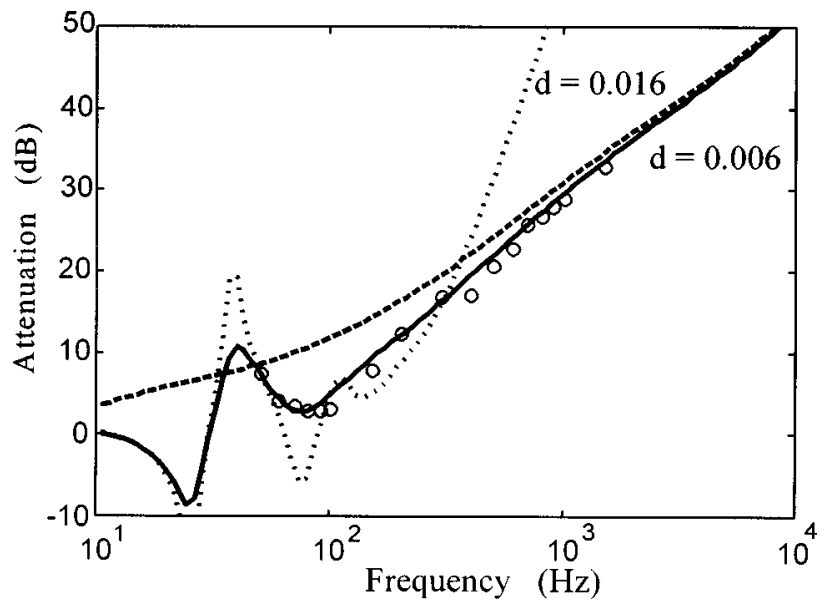

FIG. 6. Measured and computed attenuation of plane sound waves in glass wool of density $30 \mathrm{~kg} / \mathrm{m}^{3}$ and elastic modulus $16000 \mathrm{~Pa}$. The fibers are parallel to the wave front. The attenuation was found $0.20 \mathrm{~m}$ inside the glass wool sample of thickness $0.60 \mathrm{~m}$. The dotted line is computed for a volume density of fibers of 0.016 and fiber diameter $6.8 \mu \mathrm{m}$. The circles are measurements. The full line is computed for a density of 0.006 . The dashed line is calculated with the same density but assuming the fibers do not move.

\section{DISCUSSION}

The calculations of attenuation were based on a geometrical model consisting of parallel cylinders placed randomly. However, the measurements show that this model cannot be used to predict the actual attenuation when the volume density of fibers is computed from the diameters of fibers and the mass density of glass and glass wool. The measured attenuation at all frequencies can be computed if one fits the density of fibers. For the glass wool of density 14 $\mathrm{kg} / \mathrm{m}^{3}$, the volume density based on mass densities was 0.0056 and the density used to fit the data was 0.0028 . For density $30 \mathrm{~kg} / \mathrm{m}^{3}$ the volume density was 0.016 and 0.006 , respectively. Apparently real glass wool is more open than the model. A more complicated model is needed to compute all acoustic properties from the geometry of glass wool. One important property of the glass wool is the direct flow resistivity that cannot be correctly computed from the model with randomly placed, parallel cylinders.

The full line of Fig. 5 can be computed for frequencies above $100 \mathrm{~Hz}$ if one neglects the reflection of waves from the rear because the sound wave reflected from the rear side of the glass wool stack is much attenuated. A curve for attenuation without reflections is not shown in Fig. 5. The low-frequency resonance would be absent if the fibers did not move because the attenuation would be greater. The attenuation is smaller when the fibers move, because the relative velocity between air and fibers is smaller, and the energy dissipation is smaller. In Fig. 6 for the heavy glass wool, the reflections from the rear can be neglected for frequencies above $100 \mathrm{~Hz}$.

Measurements and calculations show that the attenuation is smaller when the glass wool fibers can move. In Fig. 6 one can see that a propagating sound wave in glass wool of density $30 \mathrm{~kg} / \mathrm{m}^{3}$ and frequency $100 \mathrm{~Hz}$ is attenuated $4 \mathrm{~dB}$ by a layer of thickness $0.20 \mathrm{~m}$ if the fibers move and $12 \mathrm{~dB}$ if they do not move. That means that one could increase the 
attenuation of glass wool by mechanical restriction of the movement of the glass wool.

The author's previous paper ${ }^{14}$ reported measurements of the attenuation of glass wool samples, placed in a tube of diameter $102 \mathrm{~mm}$, where they fitted tightly. These measurements could be explained by assuming the fibers did not move. For glass wool of density $40 \mathrm{~kg} / \mathrm{m}^{3}$ an attenuation of $14 \mathrm{~dB}$ for a layer of $0.20 \mathrm{~m}$ was measured at the frequency $100 \mathrm{~Hz}$. Apparently the tube prevented the movements of the fibers.

\section{APPENDIX A: CALCULATION OF THE DYNAMICAL COMPRESSIBILITY}

The dynamic compressibility was computed from the temperature increase caused by the sound pressure by the methods of the paper by Tarnow. ${ }^{8}$ The effective heat capacity of fibers per volume is called $K_{h}$. First set $K_{h}=0$, then compute the wave number of the heat wave in the air $k_{h}$ from

$$
k_{h}=\sqrt{\frac{i \omega\left(\rho c_{p}+K_{h}\right)}{\kappa}},
$$

where $\rho=1.20 \mathrm{~kg} / \mathrm{m}$ at standard atmospheric pressure 101 $\mathrm{kPa}$ and temperature $20^{\circ} \mathrm{C}, c_{p}=1.00 \mathrm{~kJ} \mathrm{~K}^{-1} \mathrm{~kg}^{-1}$ is the specific heat capacity of air at constant pressure, and $\kappa=2.57$ $\times 10^{-2} \mathrm{~W} \mathrm{~m}^{-1} \mathrm{~K}^{-1}$ is the coefficient of thermal conduction of air, both at $20^{\circ} \mathrm{C}$. $K_{h}$ is then computed from

$$
K_{h}=-\frac{2 \pi a \kappa k_{h} H_{1}^{1}\left(k_{h} a\right)}{i \omega b^{2} H_{0}^{1}\left(k_{h} a\right)} ;
$$

here $a$ is the radius of the fibers, $b^{2}$ the mean area per fiber, and $H_{0}^{1}(z)$ and $H_{1}^{1}(z)$ are Hankel functions. It is assumed that the heat capacity of the fibers is infinitely great. If it is finite, Eq. (A2) must be changed in the manner shown in an earlier paper by the author. ${ }^{8}$ To obtain self-consistency, the value of $K_{h}$ computed by Eq. (A2) is set into Eq. (A1) and a new value of $k_{h}$ is computed. This is set into Eq. (A2), etc. until the process converges to a value of $K_{h}$. This requires normally four iterations.

The dynamic compressibility of the air is computed by setting $K_{h}$ into the following equation:

$$
C=\frac{1}{P}\left[1-\frac{(\gamma-1) \rho c_{p}}{\gamma\left(\rho c_{p}+K_{h}\right)}\right] ;
$$

here $\gamma=1.40$ and $P$ is the atmospheric pressure, $101 \mathrm{kPa}$ normally.

\section{APPENDIX B: CALCULATION OF DYNAMIC RESISTIVITY}

The dynamic resistivity was also computed by a selfconsistent procedure, which is in the paper by Tarnow. ${ }^{10}$ One first chooses a value of the wave number $k_{\nu}$ of the viscous wave in the boundary layer, for example, $k_{\nu}=i / b$, then one computes the resistivity from

$$
R_{\perp}^{\prime}=\frac{2 \pi \eta k_{\nu} a}{b^{2}}\left(-k_{\nu} a+\frac{2 H_{1}^{1}\left(k_{\nu} a\right)}{H_{0}^{1}\left(k_{\nu} a\right)}\right) ;
$$

here $\eta=1.86 \times 10^{-5} \mathrm{~Pa} \mathrm{~s}$, the viscosity of air at $20^{\circ} \mathrm{C}$.
Then one computes a new value of the wave number $k_{\nu}$ from

$$
k_{\nu}=\sqrt{\frac{i \omega \rho-R_{\perp}^{\prime}}{\eta} .}
$$

A new value of $R_{\perp}^{\prime}$ is computed from Eq. (B1). After that, a new value of $k_{\nu}$ is found from Eq. (B2). This procedure is repeated until a stable value of the resistivity is obtained. This takes about four iterations.

\section{APPENDIX C: FORMULAS FOR WAVE NUMBER AND ATTENUATION}

The solution of Eq. (19) gives the wave numbers. The concentration $d$ of fibers is small, therefore the terms proportional to $d$ can be neglected, when this is done, one gets the wave number $k_{1}$ for the airborne wave,

$$
k_{1}=\sqrt{-\frac{A}{2}-\sqrt{\frac{A^{2}}{4}-B}},
$$

where

$$
A=\rho \phi C \omega^{2}+\frac{\mu \omega^{2}}{c_{11}}+\frac{i \omega R_{\perp}^{\prime}}{c_{11}}+i \omega R_{\perp}^{\prime}+i \omega R_{\perp}^{\prime} \phi C,
$$

and

$$
B=\left(\rho \mu \omega^{4}+\rho i \omega^{3} R_{\perp}^{\prime}+\mu i \omega^{3} R_{\perp}^{\prime}\right) \frac{\phi C}{c_{11}} .
$$

The wave number $k_{2}$ for the mechanical mode is

$$
k_{2}=\sqrt{-\frac{A}{2}+\sqrt{\frac{A^{2}}{4}-B}} .
$$

The attenuation is computed from the coefficients $u_{1}, u_{2}, \nu_{1}, \nu_{2}$ of Eqs. (21) and (22). Set $v_{1}=1$, and use Eqs. (20) and (23) to find the rest. The pressure can be found by setting Eq. (21) into (24). In this way

$$
p(x)=-\frac{k_{1}}{\phi C}\left[\cos k_{1} x+D \cos k_{2} x\right],
$$

where

$D=\frac{\left(\omega R_{\perp}^{\prime}\right)^{2} \cos k_{1} L}{\left(-\rho \omega^{2}+k_{2}^{2} /(\phi C)-i \omega R_{\perp}^{\prime}\right)\left(-\mu \omega^{2}+c_{11} k_{1}^{2}-i \omega R_{\perp}^{\prime}\right) \cos k_{2} L}$,

when the thickness of the sample is $L$.

The attenuation is the absolute value of the sound pressure at the free surface divided by the pressure inside the sample,

$$
\left|\frac{p(-L)}{p(x)}\right|,
$$

where $-L<x<0$.

\footnotetext{
M. D. Dahl, E. J. Rice, and D. E. Groesbeck, "Effects of fiber motion on the acoustic behavior of an anisotropic, flexible fibrous material," J. Acoust. Soc. Am. 87, 54-66 (1990).

${ }^{2}$ J. F. Allard, C. Depollier, P. Guignouard, and P. Rebillard, "Effect of a
} 
resonance of the frame on the surface impedance of glass wool of high density and stiffness,'” J. Acoust. Soc. Am. 89, 999-1001 (1991).

${ }^{3}$ R. F. Lambert, "Acoustic resonance in highly porous, flexible, layered fine fiber materials," J. Acoust. Soc. Am. 93, 1227-1234 (1993).

${ }^{4}$ R. F. Lambert, "Low-frequency acoustic behavior in highly porous, flexible, layered fine fiber materials,' J. Acoust. Soc. Am. 97, 818-821 (1995).

${ }^{5}$ M. A. Biot, "Theory of propagation of elastic waves in a fluid-saturated porous solid. I. low frequency range,' J. Acoust. Soc. Am. 28, 168-178 (1956).

${ }^{6}$ M. A. Biot, "Theory of propagation of elastic waves in a fluid-saturated porous solid. II. Higher frequency range,' J. Acoust. Soc. Am. 28, 179191 (1956).

${ }^{7}$ T. F. Johansen, J.-F. Allard, and B. Brouard, "Finite element method for predicting the acoustical properties of porous samples," Acta Acoust. 3, 487-491 (1995).
${ }^{8}$ V. Tarnow, “Compressibility of air in fibrous materials,' J. Acoust. Soc. Am. 99, 3010-3017 (1996).

${ }^{9}$ L. I. Schiff, Quantum Mechanics (McGraw-Hill, New York, 1955), Sec. 38 , p. 283.

${ }^{10} \mathrm{~V}$. Tarnow, "Calculation of the dynamic air flow resistivity of fiber materials,' J. Acoust. Soc. Am. 102, 1680-1688 (1997).

${ }^{11}$ H. Lamb, Hydrodynamic (Cambridge U.P., New York, 1993), Chap. IV, Sec. 68.

${ }^{12}$ L. D. Landau and E. M. Lifshitz, Fluid Mechanics (Pergamon, Oxford, 1959).

${ }^{13}$ J. W. S. Rayleigh, "On the influence of obstacles arranged in a rectangular order upon the properties of a medium,', Philos. Mag. 34, 481-502 (1892).

${ }^{14} \mathrm{~V}$. Tarnow, "Measurement of sound propagation in glass wool," $\mathrm{J}$. Acoust. Soc. Am. 97, 2272-2281 (1995). 